\title{
Phagocytes and the Lung
}

\author{
PETER A. WARD ${ }^{a}$ \\ Department of Pathology \\ The University of Michigan Medical School \\ M5240 Medical Science I, Box 0602 \\ 1301 Catherine Road \\ Ann Arbor, Michigan 48109-0602
}

\section{INTRODUCTION}

Activation of lung phagocytes, whether involving residential or recruited phagocytic cells (macrophages, monocytes, neutrophils), results in a series of products important for development of inflammatory injury of the lung. An understanding of the mechanisms of cell activation as well as how products of these activated cells interact with cellular and non-cellular targets to bring about tissue damage is important for the ultimate use of blocking interventions in the treatment of human inflammatory diseases. In these considerations, we will discuss cytokine products of activated macrophages, including pro-inflammatory cytokines, anti-inflammatory cytokines, oxidants, and proteinases (TABLE 1). All of the information to be presented in this paper derives from the study of an animal model of acute lung injury induced by intrapulmonary deposition of IgG immune complexes. This deposition results in a complement-dependent activation of lung macrophages, recruitment of large numbers of neutrophils, and ultimate damage of lung cells and matrix as a result of numerous products from activated macrophages and recruited (and activated) neutrophils. ${ }^{1}$ Damage in this lung model can be precisely quantitated by extravascular leakage of ${ }^{125}$ I-labeled albumin from the blood, extravasation of ${ }^{51} \mathrm{Cr}$-labeled rat $\mathrm{RBC}$, and build-up of neutrophils (as measured by lung content of myeloperoxidase (MPO) or retrieval of neutrophils from bronchoalveolar lavage (BAL) fluids). Cytokine content is usually measured in BAL fluids, although whole lung homogenates can also be employed. The IgG immune complex model may provide information relevant to an understanding of human inflammatory diseases triggered by deposition of IgG immune complexes, such as rheumatoid arthritis, systemic lupus erythematosus, vasculitis, membranous glomerulonephritis, idiopathic pulmonary fibrosis, etc.

\section{PRO-INFLAMMATORY CYTOKINES/CHEMOKINES}

There are at least three different functional categories of cytokines/chemokines as defined by their pro-inflammatory effects: Cytokines include the traditional products such as TNF $\alpha$, IL-1, interferon-gamma (IFN $\gamma$ ), etc., while chemokines include the

${ }^{a}$ Address correspondence to: Peter A. Ward, M.D., Professor and Chairman, Department of Pathology, The University of Michigan Medical School, M5240 Medical Science I, Box 0602, 1301 Catherine Road, Ann Arbor, Michigan 48109-0602. 
TABLE 1. Inflammatory Products of Lung Macrophages in Lung Injury

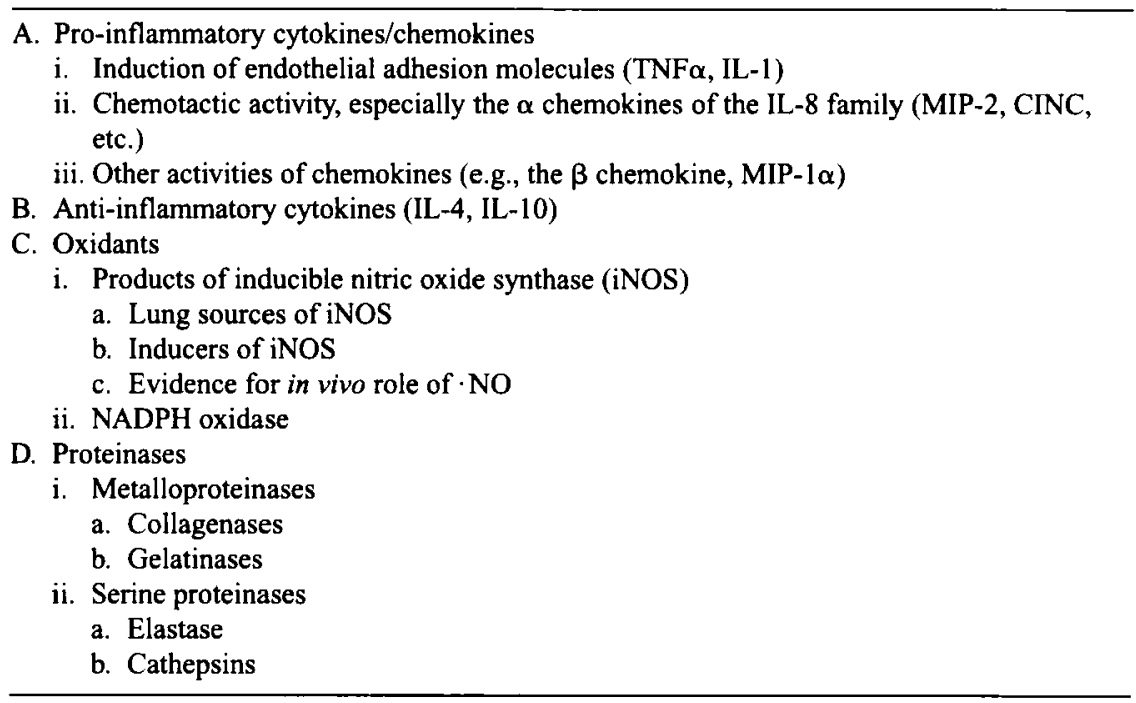

IL-8 family of $\alpha$ chemokines (chemically characterized by $\mathrm{C}-\mathrm{X}-\mathrm{C}$ linkages and including products such as IL-8; MIP-2; PF-4; GRO $\alpha, \beta, \gamma ; \mathrm{KC}$, etc.) and the $\beta$ chemokines (chemically characterized by $\mathrm{C}-\mathrm{C}$ linkages and including products such as MCP-1,2,3; MIP-1 $\alpha, \beta$; RANTES; Eotaxin; etc.). In the IgG immune complex model of acute lung injury, bronchoalveolar (BAL) fluids as a function of time contain large amounts of biologically active TNF $\alpha$ and IL-1. Although these cytokines are known to have the ability to activate macrophages and neutrophils, alone each cytokine has fairly limited in vitro activity, best described as "priming" of these cells for enhanced responses to other stimuli (e.g., cytokines, immune complexes, lipopolysaccharide, etc.). A major function of TNF $\alpha$ and IL-1 in lung appears to be induction of endothelial adhesion molecules, ICAM-1 and E-selectin, both of which are critical for the early steps required in neutrophil adhesion to the activated endothelium and eventual transmigration of neutrophils into the alveolar compartment in the inflammatory model described above. ${ }^{2}$ Blocking of TNF $\alpha$ by antibody or by soluble TNF $\alpha$ receptor-I, or blocking of IL-1 by antibody or by IL-1 receptor antagonist resulted in dramatically diminished recruitment of neutrophils and reduced development of lung injury. In the case of blockade of TNF $\alpha$, these results could be attributed to greatly reduced upregulation of lung vascular ICAM-1 as determined by the use of quantitative fixation of ${ }^{125} \mathrm{I}$-anti-ICAM-1 to the lung vasculature. Although studies with blockade of IL-1 are incomplete, the trends also suggest that IL-1 functions in this model in a similar manner to that of TNF $\alpha$. These data strongly suggest that the "early response cytokines," TNF $\alpha$ and IL-1, function in a pro-inflammatory manner by playing a key role in upregulation of lung vascular ICAM-1 and E-selectin, which are critically necessary for neutrophil recruitment.

Another functional classification of cytokines/chemokines derives from their 
chemotactic activity. ${ }^{3,4}$ Some of the $\alpha$ chemokines (IL-8 or its family relatives, such as MIP-2 and CINC, $\mathrm{KC}$ and others) and some of the $\beta$ chemokines (MIP- $1 \alpha, \beta$; MCP-1,2 and 3; RANTES; and others) are well known for the chemotactic activities, the $\alpha$ chemokine family being described as having activity chiefly for neutrophils (and to a lesser extent for $T$ cell subsets), while the $\beta$ chemokines are thought to be predominantly chemotactic for monocytes and lymphocytes. It is clear that this functional classification cannot be strictly relied upon for predictions of the in vivo role of these chemokines. In the IgG immune complex model of injury in rats, message for MIP-2 appears within $2 \mathrm{~h}$ and is maintained for the next $4 \mathrm{~h}$, followed by decline. MIP-2 protein followed a similar, albeit slightly delayed, pattern of expression. Most importantly, blocking of rat MIP-2 by antibody was significantly protective, resulting in reduced influx of neutrophils and diminishing degree of lung damage, without affecting BAL levels of TNFo. ${ }^{5}$ These data indicate that MIP-2 is important in this inflammatory model and may be functioning chiefly as a chemotactic attractant for neutrophils. A second $\alpha$ chemokine that seems to play an important role in this inflammatory model is the chemokine-induced neutrophil chemotactic factor (CINC), which is expressed in a manner similar to that described for MIP-2 (as above). Blocking of CINC similarly suppresses neutrophil recruitment and development of lung injury. ${ }^{6}$ Thus, at least two $\alpha$ chemokines play important roles in these reactions and seem to function as neutrophil chemoattractants. A third proinflammatory function of the chemokines derives from our studies in the rat lung model of IgG immune complex injury, in which MIP-l $\alpha$ expression has been demonstrated (both by mRNA and by protein) in the first 2-6 $\mathrm{h}$ of the developing reaction. Antibody-induced blocking of MIP-1 $\alpha$ results in suppressed recruitment of neutrophils and reduced leakage of ${ }^{125}$ I-albumin. In striking contrast to the protective effects of blockade of MIP-2 and CINC, blockade of MIP-1 $\alpha$ is also associated with substantial reductions in levels of TNF $\alpha$ in BAL fluids. ${ }^{7}$ This has led to the conclusion that in this model the $\beta$ chemokine MIP-l $\alpha$ functions as an autocrine regulator of TNF $\alpha$ production, the end result being enhanced upregulation of lung vascular ICAM-1 by tissue macrophages. Accordingly, as compared to MIP-2 and CINC, this chemokine appears to play a very different functional role in the inflammatory response.

\section{ANTI-INFLAMMATORY CYTOKINES}

Another functional classification of cytokines comes from the recognition that certain cytokines have very clearly been shown to have anti-inflammatory functions, especially IL-4 and IL-10. A major function of these cytokines has been suggested by their in vitro ability to suppress cytokine production by stimulated macrophages. ${ }^{8,9}$ Our employment of these cytokines in the IgG immune complex lung injury model has verified that both IL-4 and IL-10 have very powerful anti-inflammatory effects. ${ }^{10}$ When added to the airway with anti-bovine serum albumin in minute (nanogram) quantities, these cytokines are profoundly suppressive, causing dramatic reductions in albumin leak, in intraalveolar hemorrhage, and in neutrophil accumulation. Furthermore, there are profound reductions in BAL levels of TNFa, which, in turn, has been shown to result in greatly reduced lung vascular ICAM-1 expression. In the case of IL-10, in vivo blocking of this cytokine increases the level of TNF $\alpha$ in BAL 
fluids, accompanied by increased accumulation of neutrophils and intensified injury, whereas antibody to IL-4 has no effect. ${ }^{11}$ These data suggest that, in this model of lung injury, intrinsic IL-10 regulates the intensity of inflammatory damage by controlling the level of TNF $\alpha$ produced, and, correspondingly, the upregulation of ICAM-1, an essential adhesion molecule in this inflammatory model. It seems likely that there may be additional intrinsic cytokines with anti-inflammatory activity, such as IL-6 and IL-13.

\section{OXIDANT PRODUCTS OF PHAGOCYTIC CELLS}

Using the IgG immune complex model of inflammatory lung injury, evidence suggests that two oxidant-generating pathways in lung macrophages and neutrophils contribute to development of lung injury. These pathways are described in FiguRE 1. The first oxidant-generating pathway features inducible nitric oxide synthase (iNOS). This enzyme is inducible in lung macrophages by the action of three cytokines: TNF $\alpha$, IL-1, and interferon gamma (INF $\gamma$ ). In the in vivo model described above, blockade of any one of these three cytokines produces partial protection from

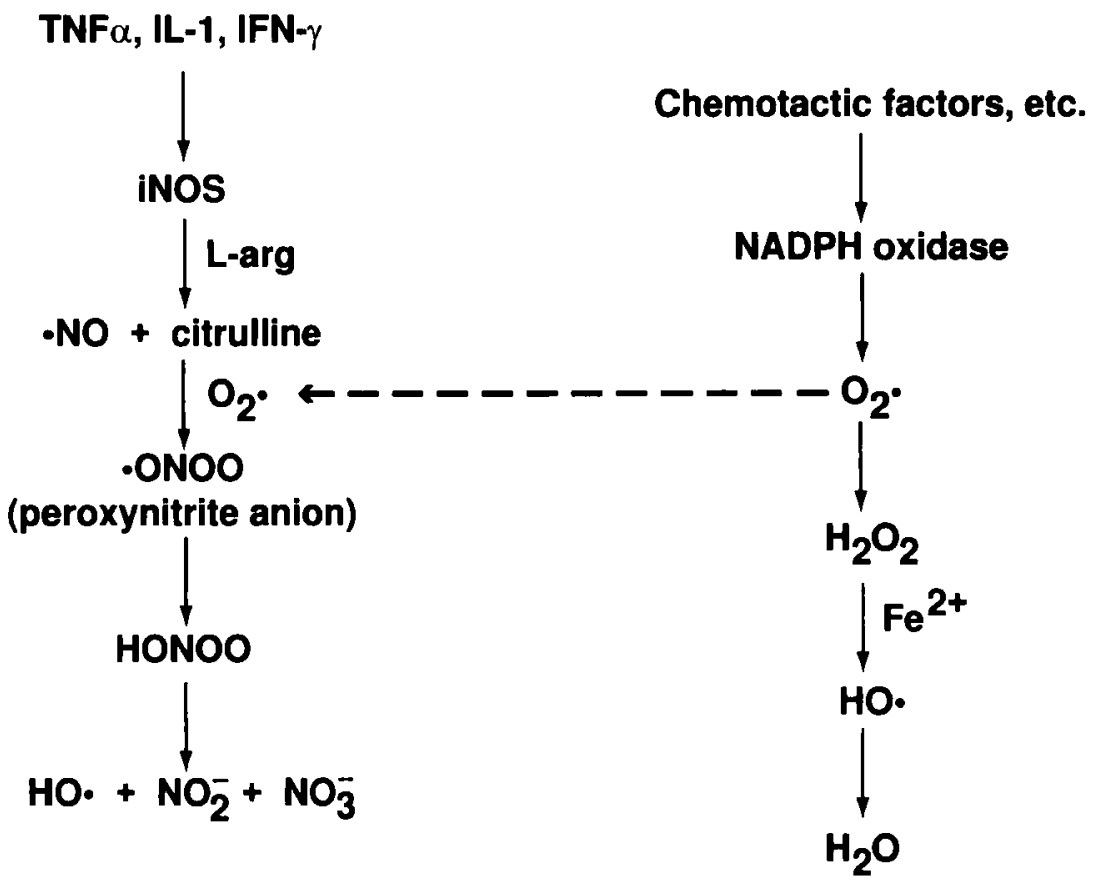

FIGURE 1. Sources of oxidants generated by activated phagocytic cells, featuring inducible nitric oxide synthase (iNOS) and NADPH oxidase. The intersection between the two pathways occurs between superoxide anion $\left(\mathrm{O}_{2} \cdot\right)$ and nitric oxide $(\cdot \mathrm{NO})$. 
lung injury and partial reduction in nitric oxide $(\cdot \mathrm{NO})$ generation (as quantitated by formation of $\mathrm{NO}_{2}^{-}\left(\mathrm{NO}_{3}{ }^{-}\right)$, whereas in vivo blockade of all three cytokines (by antibodies) almost totally suppresses $\cdot \mathrm{NO}$ production and greatly attenuates development of lung injury. ${ }^{\mathrm{I} 2}$ In the $\mathrm{NO}$ generating pathway, iNOS converts L-arginine into $\mathrm{NO}$ and citrulline. $\mathrm{NO}$ can then react with available superoxide anion $\left(\mathrm{O}_{2} \cdot\right)$ to produce the highly reactive peroxynitrite anion ( $\cdot \mathrm{ONOO})$ which, when protonated (to form HONOO) in an acidic environment can undergo hemolytic cleavage to generate the hydroxyl radical ( $\mathrm{HO}$ ), which is also highly reactive. The ultimate breakdown products of this pathway are nitrite $\left(\mathrm{NO}_{2}{ }^{-}\right)$and nitrate $\left(\mathrm{NO}_{3}{ }^{-}\right)$, which are the usual chemical surrogates for detection of $\cdot \mathrm{NO}$. Evidence for the role of the $\cdot \mathrm{NO}$ pathway in this inflammatory model was obtained by the airway instillation of analogues of L-arginine, namely, $\mathrm{L}-\mathrm{N}^{\mathrm{G}}$ monomethyl arginine and related compounds. ${ }^{13}$ Acting as competitive inhibitors of $\mathrm{L}$-arginine, these analogues in a dose-dependent manner effectively inhibit $\cdot \mathrm{NO}$ production in lung and substantially reduce development of injury. These protective effects are not associated with any reduction in neutrophil accumulation in these inflammatory reactions, suggesting that the $\mathrm{NO}$-generating pathway is a terminal effector mechanism for tissue injury. To date, there are two identified lung sources of iNOS in this model of lung injury: lung macrophages and type II alveolar epithelial cells. ${ }^{12}$ This conclusion is based not only on immunostaining for iNOS, demonstrating its presence in these two cell types, but is also based on the finding that isolation from normal lung of either alveolar macrophages or type II alveolar epithelial cells and their subsequent in vitro stimulation with IFN $\gamma$ or lipopolysaccharide (LPS) results in -NO production that is suppressible in the presence of analogues of $L$-arginine.

A second oxidase-generating system, NADPH oxidase, is present in both lung macrophages and neutrophils. ${ }^{14,15}$ Upon cell activation (with phorbol ester, immune complexes, etc.) cytoplasmic cofactors are transposed to the cell membrane to produce a protein complex that defines NADPH oxidase. This enzyme transfers single electrons, resulting in progressive reduction of $\mathrm{O}_{2}$ to $\mathrm{O}_{2} \cdot$ to $\mathrm{H}_{2} \mathrm{O}_{2}$, to $\mathrm{HO}$, and finally, to $\mathrm{H}_{2} \mathrm{O}$. NADPH oxidase may be the key source for $\mathrm{O}_{2} \cdot$ that intercalates with the iNOS pathway to react with $\cdot \mathrm{NO}$, as described above. $\mathrm{O}_{2} \cdot$ may play another important role in reducing $\mathrm{Fe}^{3+}$ in ferritin stores within cells to generate $\mathrm{Fe}^{2+}$, a reactive transitional form that can interact with $\mathrm{H}_{2} \mathrm{O}_{2}$ to facilitate electron transport, reducing $\mathrm{H}_{2} \mathrm{O}_{2}$ to $\mathrm{HO}$, thus regenerating $\mathrm{Fe}^{3+} \cdot \mathrm{H}_{2} \mathrm{O}_{2}$ can also be metabolized in the presence of chloride and MPO to generate hypochlorous acid $(\mathrm{HOCl})$, which can convert procollagenase and progelatinase into their active enzyme forms (see below). The presumed role of NADPH oxidase in this lung injury model is not only to provide $\mathrm{O}_{2}$, perhaps for the iNOS pathway (see above), but also to generate $\mathrm{HO} \cdot$ from $\mathrm{H}_{2} \mathrm{O}_{2}$, as suggested by the protective effects of catalase and the iron chelator, deferoxamine. ${ }^{16}$

\section{PHAGOCYTIC CELL PROTEINASES INVOLVED IN LUNG INJURY}

Two categories of proteinases appear to be involved in lung injury in the IgG immune complex model: metalloproteinases and serine proteinases. The most direct experimental evidence incriminating these proteinases comes from the use of in- 
hibitors. Metalloproteinases include collagenases and gelatinases, the latter having been identified in BAL fluids of animals developing IgG-immune complex-induced alveolitis. These enzymes derive chiefly from macrophages and, as the names imply, hydrolyze native or denatured collagens. Serine proteinases include elastase and cathepsins, derived both from macrophages and neutrophils. Elastolytic activity has been found in BAL fluids from inflamed rat lungs in the case of the IgG immune complex model (TABLE 2). The most compelling evidence for the role of these enzymes has been the demonstration that airway instillation of recombinant human tissue metalloproteinase inhibitor-2 (TIMP-2) or recombinant human secreted leukocyte proteinase inhibitor (SLPI) sharply reduces the intensity of injury. ${ }^{17}$ Interestingly, these protective effects are associated with reduced neutrophil accumulation, suggesting that breakdown of lung connective matrix may reduce chemotactic peptides that facilitate neutrophil recruitment under the experimental conditions of inflammation. The extent to which either macrophage-derived matrix-destroying enzymes, such as cysteine proteinases (cathepsins B, H and L), may also be involved in these lung-damaging reactions remains unknown.

\section{CONCLUSIONS}

Products of activated phagocytic cells (lung macrophages and recruited neutrophils) represent a series of toxic factors that directly or indirectly injure both lung cells and matrix. Furthermore, there is evidence for synergy between the effects of oxidants and proteinases. The interplay of cytokines/chemokines in lung inflammatory reactions triggered by intrapulmonary deposition of IgG immune complexes is complicated. On the one hand, these inflammatory reactions cause production of a series of pro-inflammatory products that facilitate upregulation of endothelial adhesion molecules, chemotactic recruitment of neutrophils, and positive autocrine stimulation of macrophages productive of more cytokine generation. Yet these inflammatory reactions are also associated with macrophage products (cytokines) that downregulate the inflammatory response. Understanding the complex biological responses could set the stage for more specific approaches for blocking of the inflammatory response in a variety of human inflammatory diseases.

TABLE 2. Evidence for Role of Proteinases in IgG Immune Complex Alveolitis

\begin{tabular}{lc}
\hline Intervention & $\begin{array}{c}\text { Reduction (\%) in Lung } \\
\text { Leak of Albumin }\end{array}$ \\
\hline rhu TIMP-2 $(0.5 \mathrm{mg})$ & 33 \\
rhu TIMP-2 $(1.0 \mathrm{mg})$ & 41 \\
rhu SLPI $(1.0 \mathrm{mg})$ & 48 \\
TIMP-2 $(1.0 \mathrm{mg})+$ SLPI $(1.0 \mathrm{mg})$ & 65 \\
\hline
\end{tabular}




\section{REFERENCES}

1. Johnson, K. J. \& P. A. WARD. 1974. Acute immunologic pulmonary alveolitis. J. Clin. Invest. 54: 349-357.

2. Mulligan, M. S., A. A. Vaporciyan, M. Miyasaka, T. Tamatani \& P. A. Ward. 1993. Tumor necrosis factor $\alpha$ regulates in vivo intrapulmonary expression of ICAM-1. Amer. J. Pathol. 142: 1739-1749.

3. SCHALl, T. J. 1991. Biology of the RANTES/SIS cytoking family. Cytokine 3: 165-183.

4. Oppenheim, J. J., C. O. C. Zachariae, N. Mukaida \& K. Matsushima. 1991. Properties of the novel proinflammatory "intercrine" cytokine family. Ann. Rev. Immunol. 9: 617.

5. Schmal, H., T. P. Shanley, M. L. Jones, H. P. Friedl \& P. A. Ward. 1996. Role for macrophage inflammatory protein-2 in lipopolysaccharide-induced lung injury in rats. J. Immunol. 156: 1963-1972.

6. Shaney, T. P., H. Schmal, R. L. Warner, E. Schmid, H. P. Friedl \& P. A. Ward. 1997. Requirement for C-X-C chemokines (macrophage inflammatory protein-2 and cytokine-induced neutrophil chemoattractant) in IgG immune complex-induced lung injury. J. Immunol. 158: 3439-3448.

7. Shanley, T. P., H. Schmal, H. P. Fried, M. L. Jones \& P. A. Ward. 1995. Role of macrophage inflammatory protein- $1 \alpha$ (MIP-l $\alpha$ ) in acute lung injury in rats. J. Immunol. 154: 4793-4802.

8. Hart, P. H., G. F. Vitti, D. R. Burgess, G. A. Whitty, D. S. Piccoli \& J. A. Hamilton. 1989. Potential anti-inflammatory effects of interleukin 4: suppression of human monocyte tumor necrosis factor alpha, interleukin 1, and prostaglandin E2. Proc. Natl. Acad. Sci. USA 86: 3803.

9. de WaAl Malefyt, R., J. Abrams, B. Bennett; C. G. Figdor \& J. E. de VRies. 1991. Interleukin 10 (IL-10) inhibits cytokine synthesis by human monocytes: an autoregulatory role of IL-10 produced by monocytes. J. Exp. Med. 174: 1209.

10. Mulligan, M. S., M. L. Jones, A. A. Vaporciyan, M. C. Howard \& P. A. Ward. 1993. Protective effects of IL-4 and IL-10 against immune complex-induced lung injury. J. Immunol. 151: 5666-5674.

11. Shanley, T. P., H. Schmal, H. P. Friedl, M. L. Jones \& P. A. Ward. 1995. Regulatory effects of intrinsic IL-10 in IgG immune complex-induced lung injury. J. Immunol. 154: 3454-3460.

12. Warner, R. L., R. Paine, III, P. J. Christensen, M. A. Marletta, M. K. Richards, S. E. WILCOXEN \& P. A. WARD. 1995. Lung sources and cytokine requirements for in vivo expression of inducible nitric oxide synthase. Am. J. Resp. Cell Molec. Biol. 12: 649-661.

13. Mulligan, M. S., J. M. Hevel, M. A. Marletta \& P. A. Ward. 1991. Tissue injury caused by deposition of immune complexes is L-arginine dependent. Proc. Natl. Acad. Sci. USA 88: 6338-6342.

14. Morel, F., J. Doussiere \& P. V. Vignais. 1991. The superoxide-generating oxidase of phagocytic cells. Physiological, molecular and pathological aspects. Eur. J. Biochem. 201(3): 523-546.

15. UMEKI, S. 1994. Mechanisms for the activation/electron transfer of neutrophil NADPH-oxidase complex and molecular pathology of chronic granulomatous disease. Ann. Hematol. 68(6): 267-277.

16. JoHnson, K. J. \& P. A. WARD. 1981. Role of oxygen metabolites in immune complex injury of lung. J. Immunol. 126: 2365-2369.

17. Mulligan, M. S., P. E. Desrochers, A. M. Chennaiyan, D. F. Gibes, K. J. Johnson \& S. J. WEIss. 1993. In vivo suppression of immune complex-induced alveolitis by secretory leukoproteinase inhibitor and tissue inhibitor of metalloproteinase-2. Proc. Natl. Acad. Sci. USA 90: 11523-11527. 